\title{
Psychiatric and Behavioral Problems in Childhood Asthma: An Opportunity for Prevention
}

\author{
Rashmi Ranjan $\operatorname{Das}^{1}\left[\right.$ [D $\cdot$ Krishna Mohan Gulla ${ }^{1}$
}

Received: 26 July 2021 / Accepted: 30 July 2021 / Published online: 11 August 2021

(c) Dr. K C Chaudhuri Foundation 2021

Asthma is a common childhood illness with a genetic basis that presents with recurrent episodes of cough, wheezing, and breathlessness. The symptoms may be episodic or persistent with flare-ups, which are caused by various triggers. One such trigger, which is less often discussed is emotional factor. Psychiatry and behavioral problems occur in an increased frequency in children with asthma [1-3]. An earlier meta-analysis found the aggregate prevalence of depressive symptoms to be $27 \%$, and anxiety symptoms to be $33 \%$ in adolescents with asthma [2]. The risk of development of depressive and anxiety symptoms was 2 times higher than the controls. A later meta-analysis included more number of studies and found $37 \%$ pooled prevalence of anxiety disorders [3]. The methods (questionnaires) used to assess the psychiatric problems varied among the studies included in these two meta-analyses, and majority of the studies were community-based. The included studies were from America, Europe, and Australia.

In this issue of the Journal, Rajhans et al. published the first study of its kind from India estimating psychiatric and behavioral problems in childhood asthma [4]. They used valid tools (M.I.N.I. KID and CBCL), which were not used in previously published studies. The overall prevalence of psychiatry morbidity was $33.3 \%$, and they identified some additional problems, including specific phobia, conduct disorder, and ADHD. However, the estimation done by the authors may not be factual, and the reasons are-the study was from a follow-up clinic, mostly controlled asthma were included, sample size was very small, and clinical diagnosis of psychiatry morbidity was not made (only questionnaire was used). In spite of these shortcomings, the data are very useful and provide important information as well as

Rashmi Ranjan Das

rrdas05@gmail.com

1 Department of Pediatrics, All India Institute of Medical Sciences, Bhubaneswar, Odisha 751019, India an opportunity for prevention of these problems in children with asthma.

The psychiatry comorbidities in asthmatic children result from various factors. A diagnosis of asthma per se puts stress on the child because there is no permanent cure, and medications are required regularly along with lifestyle modification for symptom control. These may lead to development of hypervigilance and fear in case there is frequent exacerbation or poor symptom control, finally leading to psychiatry comorbidities. In addition, children with coexistent psychiatry problems may perceive increase in symptoms leading to poor asthma control, thus setting a vicious cycle between the two. Psychiatry morbidity among the parents/caregivers also has important bearing on that of the children. In a meta-analysis, the prevalence of anxious (50\%) and depressive symptoms (44\%) were higher in caregivers of children with asthma compared to controls [5]. Anxiety and depression in the parents/caregivers have negative impact on childhood asthma increasing the severity, poor asthma control, and increase in the medication use. However, in the present study, the authors did not report the prevalence of psychiatric comorbidities among the parents/caregivers. This would have made the findings more meaningful.

We need more studies with larger sample sizes including severe or uncontrolled asthma cases from urban and rural areas to estimate the actual burden and risk factors for psychiatric morbidities and behavioral problems in children with asthma and their patents/caregivers. Till more data are available, physicians should screen children with asthma for these morbidities/problems. Studies on the effect of psychological interventions should also be conducted.

\section{Declarations}

Conflict of Interest None. 


\section{References}

1. Saragondlu Lakshminarasappa D, Chandrasekaran V, Kandasamy P. Co-morbid anxiety and depression in childhood asthma and its effect on symptom control: a cross sectional study. Pediatr Pulmonol. 2021;56:378-83.

2. Lu Y, Mak KK, van Bever HP, Ng TP, Mak A, Ho RC. Prevalence of anxiety and depressive symptoms in adolescents with asthma: a meta-analysis and meta-regression. Pediatr Allergy Immunol. 2012;23:707-15.

3. Dudeney J, Sharpe L, Jaffe A, Jones EB, Hunt C. Anxiety in youth with asthma: a meta-analysis. Pediatr Pulmonol. 2017;52:1121-9.
4. Rajhans P, Sagar R, Patra BN, Bhargava R, Kabra SK. Psychiatric morbidity and behavioral problems in children and adolescents with bronchial asthma. Indian J Pediatr. 2021. https://doi.org/10. 1007/s12098-021-03661-4.

5. Easter G, Sharpe L, Hunt CJ. Systematic review and meta-analysis of anxious and depressive symptoms in caregivers of children with asthma. J Pediatr Psychol. 2015;40:623-32.

Publisher's Note Springer Nature remains neutral with regard to jurisdictional claims in published maps and institutional affiliations. 